\title{
Signatures of Distinct Nucleosynthesis Events among Metal-Poor Stars
}

\section{Ruth Peterson*}

Astrophysical Advances

E-mail: peterson@ucolick.org

\begin{abstract}
Recently, the Peterson (2011) analysis of Mo II lines near $2000 \AA$ uncovered molybdenum overabundances of nearly a factor of ten in two mildly metal-poor turnoff stars with moderate to no $r$ - or $s$-process enhancement. Because previous analyses of two dozen metal-poor giants of similarly low $r$-process enhancement had shown only low molybdenum excesses, never exceeding a factor of three, these extreme overabundances raised the possibility that distinct nucleosynthesis events were responsible for molybdenum overproduction in these two cases. This is unexpected given the mild rather than extreme overall metal deficiency of these two stars. Here we present a progress report of determinations of molybdenum abundances based on optical Mo I lines seen in archival spectra of two dozen additional turnoff stars of similar metallicity. We conclude that distinct events do not seem to be required, for most turnoff halo field stars with $-1.8 \leq[\mathrm{Fe} / \mathrm{H}]$ $\leq-1.4$ have moderate to high molybdenum abundance excesses. Indeed, nineteen of the 28 stars presently analyzed have $+0.4 \leq[\mathrm{Mo} / \mathrm{Fe}] \leq+0.8$. We mention several ramifications of this result for early nucleosynthesis of the lightest trans-ironic elements in metal-poor stars.
\end{abstract}

XII International Symposium on Nuclei in the Cosmos,

August 5-12, 2012

Cairns, Australia

* Speaker. 


\section{Overview}

This work reports the results to date of ongoing analyses of molybdenum and ruthenium abundances in dozens of moderately metal-poor turnoff stars, based on archival Keck echelle spectra of the $3100 \AA-4000 \AA$ region. From these analyses, high molybdenum overabundances emerge frequently among mildly metal-poor field halo stars near the main-sequence turnoff. Consequently, the extremely high overabundances found by [3] for two such stars apparently do not require distinct or extremely unusual nucleosynthesis events. Because both molybdenum and ruthenium share a similar degree of enhancement in each star, but elements of lower and higher atomic number are generally less enhanced, this work confirms the conclusion of [3] that a high-entropy wind (HEW) operating in its low-entropy domain was a major source of production of these elements at the earliest times in stars whose heavy $r$-process element content is relatively low. It also confirms earlier studies, e.g. [2], that demonstrated that when overall heavy $r$-process enhancement is low, the light trans-ironic elements are overabundant with respect to the extrapolation of the standard solar $r$ process-element abundances normalized to the heavier $r$-process elements such as europium. Still puzzling, however, is why more metal-poor field halo giants do not show the excesses of a factor of three to six that are common among the turnoff stars of somewhat higher metallicities. A full report of this work is given by [4].

\section{Background}

As reviewed by [6], the relative abundances of elements heavier than iron $(\mathrm{Fe}, Z=26)$ provide interesting diagnostics of the mechanisms, sites, and environments of the nucleosynthesis processes that formed them. In single stars of metallicity below one-thirtieth solar, $[\mathrm{Fe} / \mathrm{H}]<-1.5$, elements from barium $(Z=56)$ onward are produced by rapid neutron addition on iron-peak seed nuclei in the $r$-process. Abundances of the heaviest $r$-process elements vary in lockstep, even though their overall level with respect to iron ranges over two orders of magnitude.

In contrast, the ratio of the abundances of light $r$-process to heavy $r$-process elements varies widely among metal-poor stars (e.g. [2], [5]). Both in metal-poor stars and in the Sun, many processes are invoked for the light trans-Fe elements gallium through cadmium ( $Z=31$ to 48). During the last ten years, both theoretical and observational results support as a significant source of several of these elements the low-entropy domain of a high-entropy wind (HEW) above the neutron star formed in a Type II supernova.

\section{Recent Results}

The strongest such support for HEW in metal-poor stars emerged when [3] analyzed Mo II lines near $2000 \AA$ in HST spectra of 5 turnoff stars with a mild excess of heavy $r$-process elements. HD 94028 and HD 160617, with $[\mathrm{Fe} / \mathrm{H}]=-1.4$ and -1.8 , showed extreme overabundances of the light trans-Fe element molybdenum $(Z=42),[\mathrm{Mo} / \mathrm{Fe}] \sim 1$, but lower values for $\operatorname{Zr}(Z=40)$ and $\mathrm{Ru}(Z=44)$. Neither $r$ - nor $s$-process elements were as high. Of several scenarios that synthesize nuclei in this mass range in the oldest stars, only the low-entropy regime of HEW is capable of a high excess confined to a narrow mass range. However, existing Mo I abundances for $>20$ field 
and cluster giants with $[\mathrm{Eu} / \mathrm{Fe}]<+0.6$ all show $[\mathrm{Mo} / \mathrm{Fe}]<+0.5$. This suggested that only a few distinct nucleosynthesis events produced the light trans-Fe elements in the two stars. The caveat is that the analyzed stars are all giants of lower metallicity, so $[\mathrm{Mo} / \mathrm{Fe}]$ might depend on abundance itself. Moreover, their abundance determinations relied on optical lines of Mo I; scale errors or other systematics might be involved.

\section{This Work}

This work reports the analysis of two dozen additional metal-poor turnoff stars, aimed at determining directly how unusual are the high $[\mathrm{Mo} / \mathrm{Fe}]$ and $[\mathrm{Ru} / \mathrm{Fe}]$ values of $\mathrm{HD} 94028$ and HD 160617. It is based on archival Keck HIRES echelle spectra with high resolution and high S/N that [1] obtained to measure beryllium in a hundred metal-poor stars near the main-sequence turnoff.

In 28 near-turnoff stars of similar metallicity to HD 94028 and HD 160617, the same optical Mo I and Ru I lines have been analyzed as for the giants. In the nineteen stars in which both Mo I and $\mathrm{Ru}$ I lines were detected, all have $+0.4 \leq[\mathrm{Mo} / \mathrm{Fe}] \leq+0.8$. The enhancement of heavy $r$ process elements is always modest, with $[\mathrm{Eu} / \mathrm{Fe}] \leq+0.6$. Ru always tracks Mo, but $\mathrm{Y}$ and $\mathrm{Zr}$ are always less enhanced. Mo I was not detected in any star with $[\mathrm{Fe} / \mathrm{H}] \leq-2.0$.

\section{Discussion}

That giants do not show this $\mathrm{Mo} / \mathrm{Ru}$ enhancement, despite analyses based on the same Mo I and $\mathrm{Ru} I$ lines, could be due to the lower metallicity and/or the membership in globular clusters of virtually all the low-r giants for which $\mathrm{Mo} / \mathrm{Ru}$ has been measured to date. Extending this analysis to subgiants, using both ground and space-based spectra, should assess possible nonLTE effects, and provide additional elements to constrain the HEW parameters and environment. While more metal-rich turnoff stars can be analyzed from existing archival spectra, whether lower-metallicity turnoff stars also show high $[\mathrm{Mo} / \mathrm{Fe}]$ values will require new HST $2000 \AA$ spectra.

\section{References}

[1] A. M. Boesgaard, J. A. Rich, E. M. Levesque, and B. P. Bowler. Beryllium and Alpha-Element Abundances in a Large Sample of Metal-poor Stars. Astrophysical Journal, 743:140, December 2011.

[2] S. Honda, W. Aoki, Y. Ishimaru, and S. Wanajo. Neutron-Capture Elements in the Very Metal-Poor Star HD 88609: Another Star with Excesses of Light Neutron-Capture Elements. Astrophysical Journal, 666:1189-1197, September 2007.

[3] R. C. Peterson. The Extreme Overabundance of Molybdenum in Two Metal-Poor Stars. Astrophysical Journal, 742:21, November 2011.

[4] R. C. Peterson. Molybdenum and Ruthenium in Mildly Metal-Poor Stars. submitted to Astrophysical Journal Letters, 2012.

[5] I. U. Roederer, J. J. Cowan, A. I. Karakas, K.-L. Kratz, M. Lugaro, J. Simmerer, K. Farouqi, and C. Sneden. The Ubiquity of the Rapid Neutron-capture Process. Astrophysical Journal, 724:975-993, December 2010.

[6] C. Sneden, J. J. Cowan, and R. Gallino. Neutron-Capture Elements in the Early Galaxy. Annual Review of Astronomy and Astrophysics, 46:241-288, September 2008. 